\title{
Prefacio \\ Red Internacional de Investigación Ecológica a Largo Plazo: Alcances y direcciones futuras en Chile
}

\author{
Prologue \\ International Long-term Ecological Research Network: \\ Its scope and future directions in relation to Chile
}

TERRY PARR

\begin{abstract}
Chair of ILTER, Centre for Ecology \& Hydrology, Lancaster Environment Centre, Library Avenue, Bailrigg, Lancaster, LA1 4AP, UK e-mail: twp@ceh.ac.uk
\end{abstract}

Tanto a niveles nacionales como globales existe un continuo aumento en la preocupación sobre problemas medioambientales y socioecológicos de largo plazo tales como el cambio climático, la escasez de agua, la pérdida de biodiversidad y el desarrollo sustentable. En respuesta a estas preocupaciones, tanto los políticos como los científicos y el público en general aumentaron la búsqueda de información de alta calidad sobre el medio ambiente a nivel local, nacional y global. En particular, existe un creciente interés en cómo dichos temas medioambientales afectan el bienestar humano. Igualmente importante es la necesidad de comprender cómo las actividades humanas pueden ser reguladas para proteger los servicios ecosistémicos esenciales. La sección Tema Especial en este número de la Revista Chilena de Historia Natural (RChHN) reúne ejemplos de investigaciones que pueden ayudar a satisfacer estas necesidades. Este Tema Especial se focaliza en casos concretos de estudios ecológicos y socioecológicos a largo plazo (LTER y LTSER, respectivamente, por sus siglas en inglés) en Chile, y los agrupa en el contexto del trabajo en red en el escenario internacional, particularmente en relación a la Red Internacional de Investigación Ecológica a Largo Plazo (ILTER, por su sigla en inglés).

La misión de la ILTER (http:// www.ilternet.edu) es mejorar la comprensión de los sistemas ecológicos a nivel mundial y proveer información relevante para resolver los problemas medioambientales presentes y futuros. La ILTER fue fundada en 1993, durante la Reunión Científica de Investigación Ecológica a Largo Plazo de los Estados Unidos, que se desarrolló en Estes Park, Colorado. La Red fue formada para enfrentar la creciente necesidad de comunicación global y colaboración entre investigadores con proyectos ecológicos a largo plazo. Treinta y nueve científicos y administradores representando dieciséis países participaron de dicho encuentro. Desde entonces, los programas de investigación ecológica a largo plazo se han expandido rápidamente, reflejando la creciente valorización de la investigación a largo plazo en estimar y resolver los complejos temas del medio ambiente. En agosto de 2009, la ILTER incluía como miembros a 41 redes nacionales dispuestas a su vez en cinco redes regionales, incluyendo sitios en Asia-Este del Océano Pacífico, Europa, sur de África, Norteamérica y América Central/Sur. Sin embargo, existen ecorregiones clave del mundo que todavía están subrepresentadas o ausentes, como el sur de Sudamérica. No obstante, varios países en estas regiones persiguen activamente el establecimiento de nuevas redes miembro. En consecuencia, este volumen provee una valiosa síntesis del trabajo y de los esfuerzos preliminares orientados a implementar una red de LTSER y, asimismo, provee bases claras para su incorporación en la red ILTER.

Esta naciente red chilena se caracteriza por un extenso rango latitudinal, que abarca desde los más extremos puntos del bosque templado 
de América del Sur que se encuentran en los ecosistemas semiáridos del Parque Nacional Fray Jorge $\left(30^{\circ} \mathrm{S}\right)$, pasando por los bosques lluviosos valdivianos y norpatagónicos, y las turberas de la Estación Biológica Senda Darwin en la Isla Grande de Chiloé $\left(42^{\circ} \mathrm{S}\right)$, y finalizando en los bosques subantárticos magallánicos del Parque Etnobotánico Omora $\left(55^{\circ} \mathrm{S}\right)$ en la Reserva de Biosfera Cabo de Hornos, que alberga además a los bosques más australes del planeta (Anderson et al. 2008).

A escala global, los objetivos de la ILTER son: (1) Fomentar la colaboración y coordinación entre investigadores para lograr que sean más comparables los datos de las investigaciones ecológicas y socioeconómicas a largo plazo obtenidos de todos los sitios alrededor del mundo; (2) Facilitar el intercambio, la integración, el acceso y la preservación de los datos obtenidos de los estudios a largo plazo en cada sitio; (3) Entregar información no sesgada a científicos, políticos, y al público en general, para satisfacer los requerimientos de los tomadores de decisiones en múltiples niveles; y (4) Facilitar la educación de la próxima generación de científicos enfocados en estudios de largo plazo.

Un elemento fundamental de la misión de la ILTER es informar las opciones, soluciones y decisiones pertinentes a los problemas medioambientales, proporcionando datos e información científicos relevantes para muchos de los principales problemas ambientales que enfrenta la sociedad actualmente. Algunos ejemplos de cómo la ILTER está contribuyendo en estas áreas son expuestos a continuación y muchos otros son descritos en este Tema Especial de RChHN.

\section{Cambio climático}

La ILTER y sus miembros tienen la oportunidad de jugar un papel clave en la mitigación y manejo de los riesgos generados por el cambio climático. Al integrar los resultados de sitios de investigación locales que examinan una serie de sistemas ecológicos, la ILTER puede ayudar a detectar distintas alteraciones inducidas por el clima, tanto en la biodiversidad y los ecosistemas como en los servicios que estos sostienen. Por ejemplo, está contribuyendo a la iniciativa del Programa de las Nacional Unidas para el Medio Ambiente (UNEP, por su sigla en inglés) para desarrollar una Red de Adaptación al Cambio Climático Global, cuyo objetivo es mejorar la capacidad de enfrentar las necesidades crecientes de los países para adaptar datos, conocimiento, tecnología y políticas públicas. Como miembro de esta red, la ILTER puede contribuir con una red de estaciones de campo que ayudará a construir la capacidad de adaptación de las comunidades locales y a apoyar la toma de decisiones a varios niveles de gobierno mediante el estudio de los impactos del cambio climático, evaluando opciones de adaptación y asistiendo en la transferencia de tecnologías.

Desarrollo sustentable y uso sustentable de los recursos y ecosistemas

La salud humana, la educación, y las oportunidades económicas están vinculadas a las condiciones ambientales de manera compleja. A través del trabajo conjunto de científicos de todo el mundo, la ILTER puede mejorar la comprensión de cómo los humanos interactúan con el entorno natural, cómo los ecosistemas afectan la condición humana, y cómo están relacionados el bienestar humano con la salud de los ecosistemas (Millennium Ecosystem Assessment 2005). Por ejemplo, muchas de las redes nacionales de la ILTER tienen un rol directo como sitios de demostración para la transferencia de tecnología (e.g., la Red de Investigación de Ecosistemas de China). Además, los sitios ILTER están bien posicionados tanto para beneficiar a los actores locales o regionales, como para proveer información esencial para la toma de decisiones a escala mundial.

Uso sustentable de la biodiversidad y de los servicios ecosistémicos

La diversidad de la vida sobre la tierra (biodiversidad) es uno de los principales recursos naturales de los cuales depende la sociedad humana. Las redes y sitios LTER proveen la infraestructura, las instalaciones experimentales, las bases de datos, la información y el conocimiento necesarios para un manejo eficaz y un uso sustentable de la 
biodiversidad y de los servicios ecosistémicos que esta provee. Los LTERs son requeridos para monitorear los cambios en la biodiversidad, identificar cuáles son las principales tendencias y presiones que la afectan, y evaluar los efectos de la pérdida de la misma en los procesos y servicios ecosistémicos. En todos los ecosistemas, incluyendo aquellos fuertemente modificados por las actividades humanas, dichos procesos pueden ser complicados y lentos. Por lo tanto, los enfoques a largo plazo son esenciales para proveer los datos y el conocimiento necesarios para manejar y revertir las tendencias actuales de pérdida de biodiversidad y la degradación de los ecosistemas a escalas regionales y globales. Para ayudar a abordar esta necesidad, la ILTER está involucrada en el Grupo Sistema de Observación de los Sistemas de la Tierra (GEOSS, por su sigla en inglés), cuyo objetivo es satisfacer la necesidad de información a nivel global "para respaldar a la toma de decisiones en un mundo cada vez más complejo y medioambientalmente estresado" (http://www.earthobservations.org/ about_geo.shtml). A través de GEOSS, la ILTER está real y fuertemente involucrada en el desarrollo de la Red Mundial de Observación de la Biodiversidad (http:// earthobservations.org/geobon.shtml). Es así que la ILTER y sus sitios también contribuyen a las iniciativas relacionadas con el trabajo de Evaluación del Milenio de los Ecosistemas (MEA, por su sigla en inglés); una evaluación que considera los beneficios que se obtienen de los ecosistemas, y cómo los cambios en estos servicios ecosistémicos han afectado el bienestar humano. La ILTER actualmente sintetiza la información sobre cómo los servicios ecosistémicos están cambiando en sus sitios de estudio y las causas y consecuencias de estos cambios. Esta información será utilizada en las nuevas evaluaciones regionales de ecosistemas y también en una evaluación a nivel mundial, y en iniciativas relacionadas, tales como "La Economía de los Ecosistemas y la Biodiversidad” (TEEB, por su sigla en inglés).

\section{Riesgos y desastres ambientales}

Los sitios ILTER no son inmunes a los peligros y las catástrofes (sequías, inundaciones, pestes y brotes de enfermedades y tifones) que regularmente aparecen en los titulares de los diarios. Sin embargo, la naturaleza a largo plazo del trabajo y nuestra capacidad para realizar estudios comparativos entre varios sitios, permiten a la ILTER poner estos eventos en su contexto espacial y temporal, y generar las bases que permitan entender estos eventos y adoptar acciones para responder a los mismos. Algunos buenos ejemplos al respecto son el estudio comparativo sobre el impacto de los tifones y huracanes en diferentes partes del mundo y las investigaciones sobre brotes de enfermedades y sus causas, tales como el estudio sobre el rol de las poblaciones de roedores en la propagación del hantavirus en Nuevo México (Yates et al. 2002).

En todos estos ámbitos, la ILTER ha reconocido la necesidad de incluir la dimensión humana del cambio ambiental, y para lograrlo trabaja cada vez más con científicos sociales, economistas y comunicadores de la ciencia. En algunos países, se han desarrollado sitios LTER urbanos, mientras en otros sitios más grandes es común la incorporación de procesos sociales y económicos y la participación activa de los interesados en la investigación (e.g., la Red Finlandesa de Estudios Socio-Ecológicos a Largo Plazo FinLTSER, http:// www.environment.fi/syke/lter, y la US LTER en la ciudad de Baltimore, http:// www.beslter.org).

\section{NECESIDADES APREMIANTES}

Una parte fundamental de la estrategia actual de la ILTER es aumentar el potencial de su aproximación a través del entrenamiento, el desarrollo de asociaciones estratégicas y la adición de nuevos sitios y redes como miembros. Aunque la ILTER actualmente cuenta como miembros con más de 40 redes nacionales, su cobertura del globo está lejos de ser completa, y la ILTER está comprometida con el objetivo de expandirse mediante la adición de sitios y redes que suman su valor intrínseco y ayudan a responder preguntas científicas críticas, motivadas por la investigación. Es de particular importancia la necesidad de mejorar la cobertura de los sitios 
en el hemisferio sur, principalmente en África y América del Sur.

Claramente, una red LTSER en Chile constituiría un valioso aporte a la capacidad global de la ILTER. Como se estableció anteriormente, Chile es un país extraordinario, ya que abarca un gran rango latitudinal y altitudinal, e incluye una alta diversidad de hábitats y servicios ecosistémicos (Armesto et al. 1998). Este Tema Especial de RChHN provee excelentes ejemplos sobre iniciativas LTSER en Chile y describe la motivación para el establecimiento de una nueva red nacional de investigaciones a largo plazo. Además de registrar continuamente datos ambientales de alta calidad (e.g., Gutiérrez \& Meserve 2003, Meserve et al. 2003), la red chilena de LTSER proporcionará la infraestructura necesaria para conducir investigaciones internacionales $\mathrm{e}$ interdisciplinarias, e innovadores cursos de campo que integren la ecología, la filosofía ambiental y otras disciplinas (Rozzi et al. 2008). Se espera que la red chilena de LTSER también fortalezca los vínculos entre académicos y actores locales y regionales, facilitando la integración entre la ciencia y la sociedad. Como hemos visto a través de varios artículos en este Tema Especial de RChHN, Chile provee de otro ejemplo de cómo incluir el componente social (la "S") en LT(S)ER con el fin de asegurar que la investigación sea cada vez más relevante para los asuntos sociales. La emergente red chilena de LTSER y sitios asociados merecen todo el éxito posible, y sin dudas será un valioso aporte a la creciente familia de sitios y científicos que constituyen la ILTER.

\section{MATERIAL COMPLEMENTARIO}

La versión en inglés de este artículo está disponible como Material Complementario online en http://rchn.biologiachile.cl/ suppmat/2010/1/MC_Parr_2010.pdf

\section{LITERATURA CITADA}

ANDERSON CB, GE LIKENS, R ROZZI, JR GUTIÉRREZ, JJ ARMESTO \& A POOLE (2008) Integrating science and society through longterm socio-ecological research. Environmental Ethics 30: 295-312.

ARMESTO JJ, R ROZZI, C SMITH-RAMÍREZ \& MTK ARROYO (1998) Effective conservation targets in South American temperate forests. Science 282: 1271-1272.

GUTIÉRREZ JR \& PL MESERVE (2003) El Niño effects on soil seed bank dynamics in north-central Chile. Oecologia 134: 511-517.

MESERVE PL, DA KELT, WB MILSTEAD \& JR GUTIÉRREZ (2003) Thirteen years of shifting top-down and bottom-up control. BioScience 53: 633-64.

MILLENNIUM ECOSYSTEM ASSESSMENT (2005) Ecosystems and human well-being: Synthesis. Island Press, Washington, DC, World Resources Institute. (en línea) URL: http:// www.millenniumassessment.org/en / Reports.aspx.

ROZZI R, JJ ARMESTO \& R FRODEMAN (2008) Integrating ecological sciences and environmental ethics into biocultural conservation in South American temperate subAntarctic ecosystems. Environmental Ethics 30: 229-234.

YATES T, J MILLS, C PARAMETER, T KSIAZEK, R PARAMETER et al. (2002) Biocomplexity and hantavirus pulmonary syndrome: The ecology of an outbreak. BioScience 52: 989-998. 